\title{
Transcranial Magnetic Stimulation for Major Depression in the Elderly: a Systematic Review and Meta-analysis
}

Ana Maia ${ }^{1-3^{*}}$, Leandro Valiengo $0^{4,5^{*}}$, Gonçalo Cotovio ${ }^{1-3}$, Pedro Gordon ${ }^{5}$, André R. Brunoni ${ }^{4,5}$, Orestes V. Forlenza ${ }^{4 \#,}$ Albino J. Oliveira-Maia'-3\#

1. Champalimaud Research and Clinical Centre, Champalimaud Centre for the Unknown, Lisbon, Portugal; 2. Department of Psychiatry and Mental Health, Centro Hospitalar de Lisboa Ocidental, Lisboa, Portugal; 3. NOVA Medical School | Faculdade de Ciências Médicas, Universidade Nova de Lisboa, Lisboa, Portugal; 4. Laboratório de Neurociências (LIM-27), Departamento e Instituto de Psiquiatria, Hospital das Clínicas HCFMUSP, Faculdade de Medicina, Universidade de São Paulo, SP, Brasil; 5. Serviço Interdisciplinar de Neuromodulação (SIN), Departamento e Instituto de Psiquiatria, Hospital das Clínicas HCFMUSP Faculdade de Medicina, Universidade de São Paulo, SP, Brasil * these authors contributed equally to this work; \# these authors contributed equally to this work.

\section{INTRODUCTION}

Major Depressive Disorder (MDD) and depressive symptoms in the elderly are a serious public health concern, with an estimated prevalence of $1-4 \%$ and $8-16 \%$, respectively ${ }^{1}$. However, Treatment Resistant Depression (TRD) is common among the elderly, with an estimated rate of 28.9 per 100 person-years². Repetitive Transcranial Magnetic Stimulation (rTMS) is a non-pharmacological intervention approved for the treatment of $M D D$, being a valuable alternative for patients with $\mathrm{TRD}^{3}$. However, the value of this intervention in the elderly remains unknown ${ }^{4}$. In this study, we intend to assess the effectiveness of rTMS for the treatment of MDD in the elderly.

\section{METHODS}

First, a systematic review was performed, regarding efficacy of rTMS as a treatment for MDD among elderly patients. Secondly, random-effects meta-analyses using standardized mean differences (SMD) were conducted to assess primary continuous outcome [change in Hamilton Depression Rating Scale (HDRS) score], while odds-ratio (OR) were used to assess secondary categorical outcomes (response and remission).

\section{RESULTS}



Flow diagram

15 included studies in quantitative synthesis Total of 326 MDD patients and 290 healthy controls

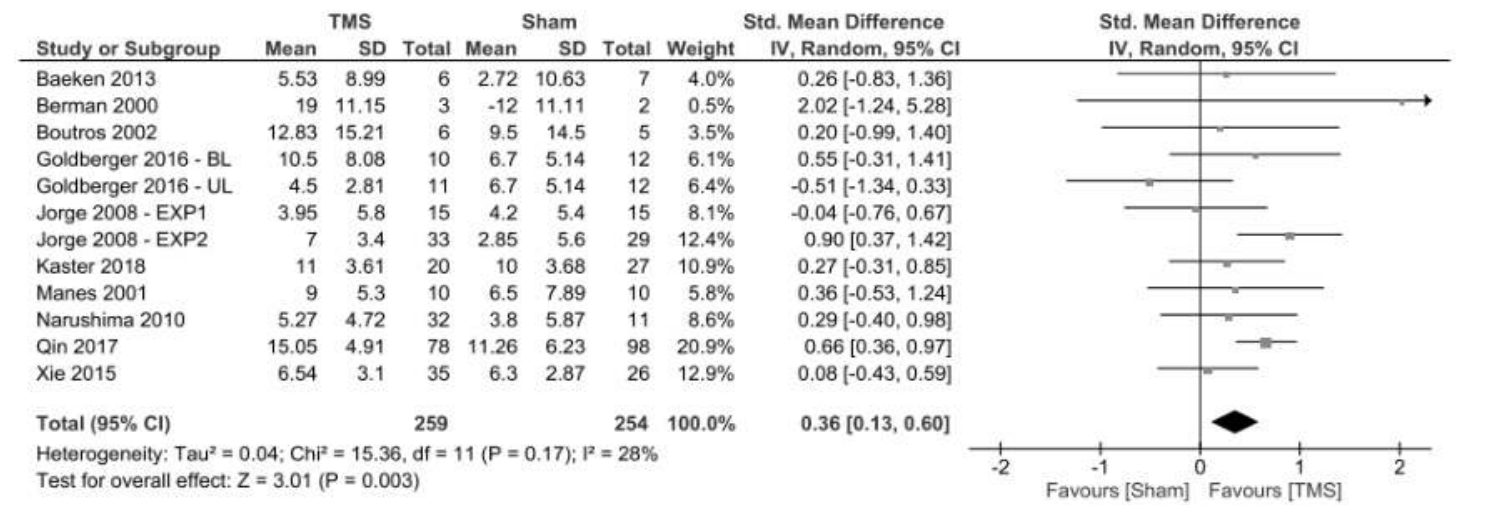

Forest plot for primary outcome HDRS reduction

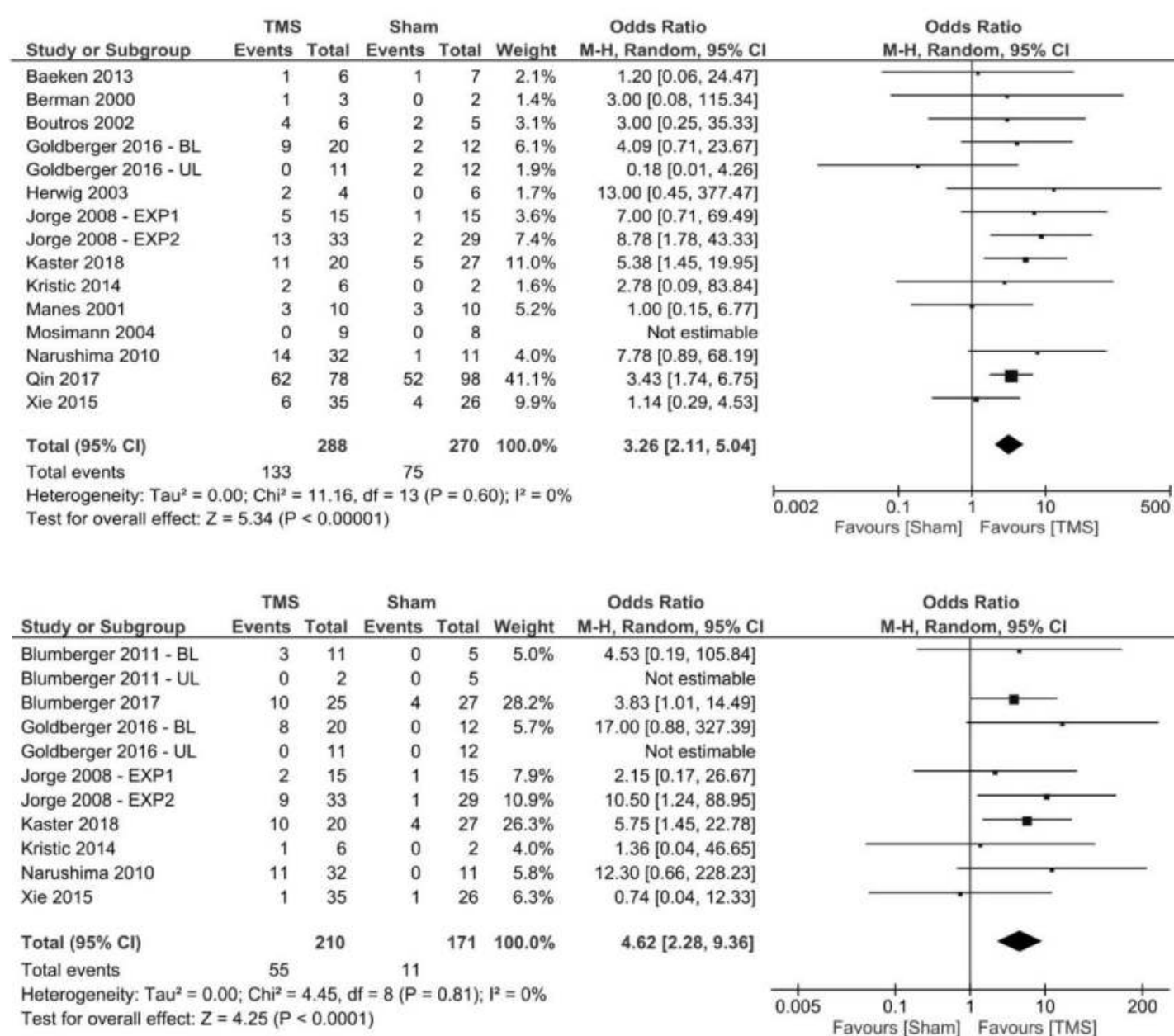

Forest plot for secondary outcome Clinical response

Forest plot for secondary outcome Clinical remission

\section{DISCUSSION AND CONCLUSIONS}

Our results support that rTMS has a significantly higher antidepressant effect than sham treatment, with a SMD of $0.36(95 \% \mathrm{Cl}=0.13$ to 0.6$)$ in continuous outcome and an OR of $3.26(95 \% \mathrm{Cl}=2.11$ to 5.04$)$ and $4.62(95 \% \mathrm{Cl}=2.28$ to 9.36 ) in categorical outcomes (response and remission rates, respectively).
Despite the paucity of data and the heterogeneity of studies, our results support that rTMS is an effective and safe treatment for MDD in the elderly, and that it should be considered in the treatment of this vulnerable and prevalent population. 